resulting in avoidable admissions and prolonged hospital stays. They went on to describe how hospice at home services could have a significant impact in enabling patients to die in their own home. The existing night hospice at home service provided practical support to patients and carers between $10 \mathrm{pm}$ and $8 \mathrm{am}$. Building on the 7 C's of the Gold Standards Framework (2005) the team promoted contingency planning and service co-ordination ensuring that they were already an integral and valued part of the community based services.

However, with an expected increase in the number of deaths from cancer and non-cancer deaths (Kings Fund 2011) and a local emphasis on preventing unnecessary admissions (Health and Well-being Board, 2012) a wide ranging review of the hospice at home service was undertaken in 2012. Service users and health care colleagues emphasised the importance of:

1. A $24 / 7$ service

2. Rapid response

3. Not being reliant on external funding

4. Supporting carers via 'on the job training and support'

5. Being accessible

6. Highly skilled, consistent and compassionate staff

The review also identified several unmet needs which could be addressed by an expanded hospice a home team, these included:

- Crisis intervention, for example whilst acute symptoms were addressed, carer stress or ill health

- Rapid response and extended packages of care for changing needs

- Pre-admission to the hospice in-patient unit

- Supported discharge from the hospice in-patient unit

- Rapid discharge from the acute sector

Our service outcomes include:

- Provision of a hospice at home service tailored to meet local needs

- Increased integration of hospice services.

- Cost effective use of hospice resources

- Increasingly achieving preferred place of care/death

\section{P13 EVENING CALL}

Eirian Levell. St Wilfrid's Hospice, Eastbourne, United Kingdom

\subsection{6/bmjspcare-2013-000591.35}

Introduction The End of Life Care (EoLC) Strategy (2008) and Preferred Priorities of Care (2011) documents support enabling people to remain in their own homes.

Prompted by the volume of calls into the in-patient unit, and workload of the district nurses in the early evening, the hospice piloted an extension to the Hospice@Home (H@H) working hours.

Aim To reduce unnecessary admission to hospital at the end of life by providing extended access to support and guidance for patients, carers and health care professionals.

Method Following a three month analysis of telephone advice calls received by the Inpatient Unit (IPU), outside of $\mathrm{H} @ \mathrm{H}$ working hours, the pilot took place over a 9 month period. This extended the accessibility of trained nurse contact from $4.30 \mathrm{pm}$ to $9 \mathrm{pm}$ on a daily basis.

Results Throughout the pilot phase, calls were recorded and outcomes summarised, paying particular attention to the purpose of calls, caller identity, and the time the call was received. These statistics provided evidence that the service was not routinely accessed after $7 \mathrm{pm}$.

During the pilot, five admissions to hospital were prevented, primarily due to the swift response and symptom management by $\mathrm{H} @ \mathrm{H}$. Patients and carers benefitted from the flexibility and accessibility of a trained nurse to action changes, provide continuity and reassessment from $8.30 \mathrm{am}$ until $9 \mathrm{pm}$.

There were a high proportion of calls requesting complex symptom advice, reporting family distress and requesting an increase in care.

Conclusion Staffing the extension to the service proved challenging however the pilot was positively evaluated by the district nursing team.

Guidance, reassurance and prompt response to need, undoubtedly contributed to the prevention of crisis admissions to the acute trust.

\section{\begin{tabular}{|l|l}
\hline P14 THE IMPACT OF AUDIT ON CNS TEAM DEVELOPMENT \\
\hline
\end{tabular}}

Vanessa Gibson, June Patel. St Richards Hospice, Worcester, Worcestershire

\subsection{6/bmjspcare-2013-000591.36}

In 2011 the Hospice Clinical Nurse Specialist (CNS) team were facing a significant increase in patient referrals numbers (approximately 70 per month). The demand for community based care, rather than acute hospital admission, was also resulting in increasingly complex and urgent referrals from all areas of the local healthcare economy. In addition, palliative and end-oflife care services traditionally available only to cancer patients were increasingly being accessed by patients with non- malignant diagnoses.

In response the CNS team undertook a month long audit of referrals to the community nurse specialist service. The audit revealed:

1. Low levels of referral information, resulting in delayed contact

2. Difficulties assessing levels of urgency

3. Lack of clarity regarding other services involved with the patient

4. Unnecessary home visits

As a result the CNS team has now introduced the following:

1. A new streamlined referral process

2. A full time specialist triage nurse who makes first contact with all referrals:

She:

- Responds to all referral agencies

- Assesses level of urgency

- Holds her own low-level caseload that she contacts by phone

3. Helpline established and available to patients and health care professionals: this service provides immediate access to a highly skilled CNS

4. Accessing and completing the electronic notes systems to ensure comprehensive and up to date clinical information is readily available to the wider healthcare team.

5. Enhanced patient documentation

Continuous development is key and plans are being developed for: 
- A visiting 'rapid response' CNS

- A nurse led triage clinic

- Increased triage nurse hours

The CNS team have used audit to truly 'hear' what is happening in practice and as a result have used this knowledge to lead service redesign- a key aspect of the CNS role identified by National Cancer Action Team (2010)

\section{P15 THE RIGHT WAY TO WRITE? DEVELOPMENT OF NEW DOCUMENTATION FOR COMMUNITY PALLIATIVE CARE}

Hilary McPolin, Mary McMullan, Grainne McGinnity, Fiona Flynn. Northern Ireland Hospice, Belfast, N. Ireland

\subsection{6/bmjspcare-2013-000591.37}

Background/Context Efficient, comprehensive documentation not only provides a record of care, but also reflects the quality of that care whilst reinforcing care standards. However, documentation in palliative care has often fallen short of these ideals (McEvoy, 2000). Following the development of a centralised triage system for new referrals to a community palliative care service, it was recognised that there was duplication of information being recorded by both the triage staff and the community nurses. There was also a general consensus that the triage documentation should be incorporated into the nursing assessment tool to reflect the patients' journey and promote continuity of care.

Aim To develop user friendly, time efficient documentation that provides a comprehensive holistic assessment of specialist palliative care patients and their families.

Approach Used A project team was formed with representatives from the triage team and the community nursing teams. Following a review of in-house documentation, Liverpool Care Pathway (LCP) and assessment tools from both hospital and community palliative care teams, a new tool was developed and piloted. Minor changes were made, based on the initial evaluations and subsequently, a second pilot was undertaken before the tool was finalised.

Outcomes The new tool was rolled out to all the community teams in January 2013. Feedback to date suggests a reduction in both the amount of duplication as well as the amount of time spent on recording the required information.

Application to Hospice Practice The new documentation tool reflects a holistic, comprehensive, specialist palliative care nursing assessment, in a user friendly, time efficient format. It has improved communication and accessibility of information and recognises the need to increase the capacity for recording the needs of carers. Better documentation can lead to better patient care.

\section{P16 PLANNING YOUR WEEK: A NEW MODEL FOR RURAL WORKING}

Derek Hart, Cathy Martin, Maggie Draper. St Luke's Hospice Plymouth, Plymouth, United Kingdom

\subsection{6/bmjspcare-2013-000591.38}

An examination of caseload management is currently being undertaken by 2 community palliative care nurses who cover a large area, encompassing the environs of city to remote moor land. The remote areas have no mobile telephone access and poor infrastructure.
The caseload was analysed and divided into 7 geographical areas (zones). Visits were to be arranged to these zones on specific days to optimise efficiency, taking into account $\mathrm{mdt}$ commitments.

The aims were:

- To increase efficacy of visits, reduction of interruptions

- Reduce travelling time and mileage

- Improve response time from referral to first visit,

- Improve reliability and therefore develop stronger integrated working.

- Improve wellbeing and support of the CNS.

- Move from reactive "fire fighting" to proactive care where crises were predicted where possible.

The approach used was:

- A time table of areas and days was created.

- Two nurses covered the caseload of approx 60 patients.

- The timetable took into account mentorship needs of the new CNS. It promoted men- tee and mentor working in neighbouring zones. Meeting places in the field (literally!) were highlighted should support be required.

- 1 nurse per day was allocated to triage calls and trouble shoot.

- The administrative team were involved in planning and were aware of who to call.

Outcomes - to be measured / reviewed September 2013

- Supportive mentorship of new CNS in rural environment

- Reduced Mileage

- Reduced interruption from mobile phone calls.

- Reduced sickness

- Improved record keeping. The two nurses were reliant on each others records to provide continuity of care.

- A more efficient use of laptops reducing the need to return to base.

- Development of professional relationship between nurses encouraging peer review.

- Improved relationships with GPs and community nursing teams

\section{P17 THE COMMUNITY AND HOSPICE HOME NURSING SERVICE}

${ }^{1}$ Lesley Spencer, ${ }^{2}$ Karen Masetti, ${ }^{3}$ Gina Starnes. ${ }^{1}$ Princess Alice Hospice, Esher, England, ${ }^{2}$ Central Surrey Health, Ewell, England, ${ }^{3}$ St Catherine's Hospice, Crawley, England

10.1136/bmjspcare-2013-000591.39

Background/ Context End of Life care support throughout Surrey had been fragmented and lacked an integrated approach. As a result three organisations came together to form the 'Community and Hospice Home Nursing Service'.

The service supports a multi-disciplinary partnership approach to delivering nursing care at home. Central Surrey Health (CSH), and two Independent Hospices; Princess Alice Hospice (PAH) and St Catherine's Hospice ( $\mathrm{StCH}$ ) are now delivering a seamless seven day, 24 hour service.

Main Aim To provide care that avoids inappropriate hospital admissions and enables more people to be cared for and die in their place of choice. 